\title{
Assessing professional and clinical competence: the way forward ${ }^{\dagger}$
}

\author{
Nick Brown \& Monica Doshi
}

\begin{abstract}
Recent developments in postgraduate medical education for the training of junior doctors in the UK necessitate changes in all parts of the curriculum, including the assessment system. There is a move away from the limited, traditional one-off assessment towards multidimensional, broader assessments of a doctor's longer-term performance. This is accompanied by the rapid development of assessment tools, collectively termed workplace-based assessments, and is in keeping with an outcome-based approach to medical education and its increasing professionalisation. In addition to clinical skills, other aspects of being a good practitioner are being assessed, including team-working, working with colleagues and patients, probity and communication skills. Using a combination of tools gives the assessment process high validity. Of the many challenges posed by these changes is the need for data on their reliability in psychiatry. There must be a clear process for applying assessments, national standardisation and training for those using asessment tools.
\end{abstract}

Assessments (Boxes 1 and 2) are an essential and integral part of medical education. Assessments enable us to make decisions about trainees - whether and how much they have learnt and whether they have reached the required standard (Rowntree, 1987). They can influence the way a student learns (Broadfoot, 1996), the motivation a student has for learning and the content of what is learnt. For an assessment method to be acceptable it needs to be valid, reliable, practical and have a positive effect on a trainee's learning (Newble \& Cannon, 2001).

Poorly selected assessment methods can lead to passive or rote learning (to get through an exam ination), which is associated with a rapid decay of knowledge and sometimes an inability to apply it in real situations (Stobart \& Gipps, 1997). The main components of undergraduate and postgraduate assessment in medicine have traditionally been written and clinical examinations. These have strengths and weaknesses. The majority are now better in an all-round sense in that they test a number of dimensions, for example knowledge, problem-solving and communication skills, but by definition they are still one-off assessments. A single, sometimes non-clinical, event establishes

${ }^{\dagger}$ For a commentary on this article see pp. 89-91, this issue.

\begin{tabular}{|c|c|}
\hline CBD & Case-based discussion \\
\hline CEX & Clinical evaluation exercise \\
\hline DOP & Directly observed procedure \\
\hline Mini-CEX & Mini-clinical evaluation exercise \\
\hline MSF & Multisource feedback \\
\hline OSATS & $\begin{array}{l}\text { Objective structured assessment } \\
\text { of technical skills }\end{array}$ \\
\hline OSCE & $\begin{array}{l}\text { Objective structured clinical } \\
\text { examination }\end{array}$ \\
\hline RITA & Record of in-training assessment \\
\hline SP & Standardised patient \\
\hline WPA & Workplace assessment \\
\hline
\end{tabular}

what a doctor knows (e.g. in a multiple choice paper), knows how to do (e.g. in a short-case viva or extended matching questions) or is able to show how to do 'on the day' (e.g. in a long case or an objective structured clinical examination, an OSCE). They are basic tests of competence, not assessments of day-to-day performance. They specifically do not assess other attributes necessary for a person to perform consistently well as a doctor, for example team-working skills.

Nick Brown is a consultant in general adult psychiatry (Lyndon Resource Centre, Hobs Meadow, Solihull B92 8PW, UK. E-mail: nicholas. brown@bsmht.nhs.uk), Clinical Sub-Dean at the University of Birmingham and a Sub-Dean of the Royal College of Psychiatrists. He chairs the West Midlands Regional Training Committee and the Royal College of Psychiatrists' Standing Advisory Committee on Basic Training. Monica Doshi is a part-time consultant in general adult psychiatry and an honorary senior lecturer and Phase II Assessment Coordinator at the Joint Leicester Warwick Medical Schools. 


\section{Box 2 Useful definitions of educational terms related to assessment \\ Assessment \\ A systematic process of collecting and interpreting information about an individual in order to determine their capabilities or achievement from a process of instruction \\ Formative assessment Occurs during the teaching process and provides feedback to the trainee for their further learning \\ Summative assessment Occurs at the end of the learning process and assesses how well the trainee has learnt \\ Criterion-referenced assessment \\ The pass mark is determined against achievement of a predetermined \\ Norm-referenced assessment standard, for example those who score over $65 \%$ will pass \\ The pass mark is determined by a trainee's performance compared with that of others, for example the top $45 \%$ of students will pass \\ Appraisal \\ An on-going process designed to highlight the strengths and weaknesses of a person in order to encourage their own professional development and their contribution to the development of the organisation in which they work \\ Evaluation \\ The process of collecting and interpreting information about an educational process in order to make judgements on its success and to make improvements (note: in the USA this term is sometimes used to mean assessment, as defined above) \\ Validity \\ The degree to which a test measures what it is intended to measure, for example whether the content of an assessment covers the content of the syllabus (content validity) and whether a test predicts the future performance of a student (predictive validity) \\ Reliability The degree to which one can depend on the accuracy of a test's results, for example whether different examiners would award the same marks given the same candidate performance (interrater reliability) or whether the same set of students would score the same marks if the test were repeated with them under the same conditions (test-retest reliability)}

\section{The outcome-based approach}

Current developments in medical education and training dictate the necessity and give the opportunity for significant change in all aspects of the assessment system. Changes that have occurred in undergraduate training in the decade or so since the publication of the first edition of Tomorrow's Doctors (General Medical Council, 1993) are now being followed by similar initiatives in postgraduate education.

The current shift in medical education is towards outcome-based learning. Outcome-based education can best be summed up as results-oriented thinking. It is the opposite of input-based education, where the emphasis is on the educational process, almost regardless of the result. In outcome-based education, the outcomes agreed for the curriculum guide what is taught and, importantly here, what is assessed. Exit outcomes, i.e. the doctor's performance capabilities, are therefore a critical factor in determining both what is to be learnt (the curriculum) and what is to be tested (the assessments). Assessment programmes in outcome-based education must be systematic and include criteria for each of the abilities defined.

The focus is on the care delivered to different patients and in different settings.

Newer assessment methods need to be able to assess what a doctor actually does in everyday practice (Miller, 1990), rather than one-off assessments of what they 'know', 'know how' or can 'show how' (Fig. 1). This requirement has led to the rapid development of suitable assessment tools and schedules, generally brought together under the title of workplace assessments.

Workplace assessment is one of the cornerstones of the emergent Postgraduate Medical Education and Training Board (PMETB; http://www.pmetb. org.uk/), whose vision includes the rapid expansion of such assessments and their incorporation into a strategy that links assessment in the workplace with examinations of knowledge and clinical skills throughout training. PMETB states within its principles that assessment(s) must be fit for 


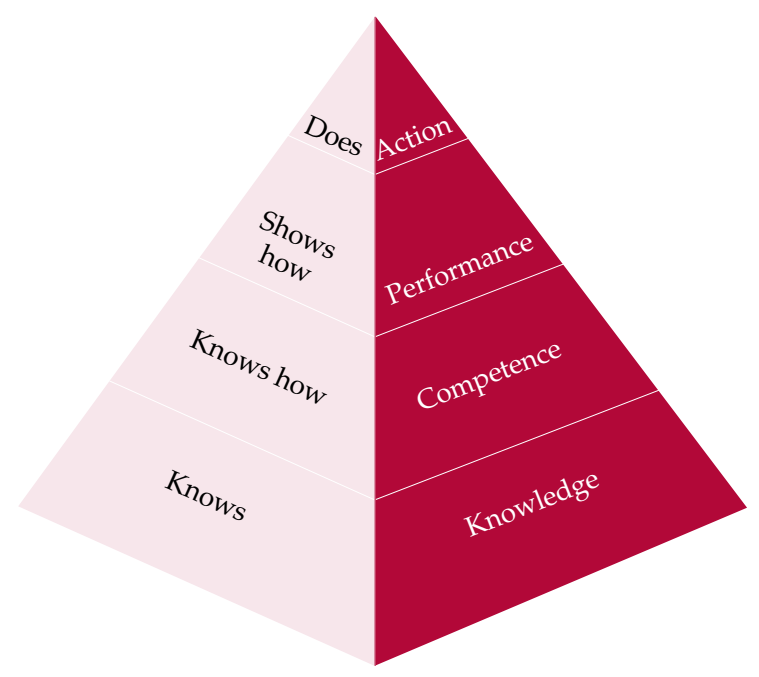

Fig. 1 Miller's framework for assessment (after Miller, 1990, with permission).

purpose, based on curricula, mapped to a blueprint of curricula content and use methods that are valid, reliable, psychometrically supported and in the public domain (Postgraduate Medical Education and Training Board, 2004a).

All assessment systems and, indeed, curricula must now satisfy PMETB's published requirements (Postgraduate Medical Education and Training Board, 2004a,b).

\section{Workplace assessment}

Workplace assessment (inevitably abbreviated to WPA) taps into the potentially best way of collecting data on trainees' performance: personal witnessing of their interactions with patients over a period of time. It has the advantage of high validity precisely for this reason. However, it is not feasible to monitor every interaction every day, hence the need to develop efficient and effective techniques for optimising the process.

To ensure that the right competencies are learnt and validly and reliably assessed, workplace assessments in psychiatry, as in medicine in general, need to be considered alongside the development of curriculum models. The clear advantages of assessment in the workplace include the opportunities for feedback and the educational value conferred by putting emphasis on 'real-time' assessment. It is a strange fact of medical life that trainees are infrequently formally observed in daily encounters with patients. This limits the opportunity for continuous and developmental feedback on clinical performance. The introduction of assessment in the workplace means that far greater emphasis can be placed on patient-based learning and far more detailed, valid and reliable observations of doctors' clinical performance can be obtained.

It is well documented that assessment drives learning (Broadfoot, 1996). Thus, if Dr A, a senior house officer, is assessed only by a multiple choice paper, she is likely to direct her learning towards facts from books. If she is assessed in the workplace, she is more likely to focus her learning on patientbased activities.

\section{Benefits of multiple assessments and assessment methods}

In an ideal system the trainee is tested by multiple raters using different tools repeatedly over a period of time. Clearly, no single rating is able to provide the whole story about any doctor's ability to practise medicine, as this requires the demonstration of on going competence across a number of different general and specific areas. Patterns emerge when an individual is subject to many assessments, thus diminishing problems of sampling and interrater reliability.

A further important reason for using different types of assessment is the specificity of the various assessment tools themselves. The development of a competency-based approach to training sets the most challenging agenda of accurately assessing a student both in individual components of competency and in overall performance.

Much of the existing data on the use of workplacebased assessments is derived from other medical specialties and often from other countries (Norcini et al, 1995). In psychiatry, we must develop or adapt existing assessment methods and assessors in order to produce accurate and defensible data.

The strategy of using multiple assessments could quickly become burdensome, since the gold - you might say platinum - standard would be one of infinite measurements applied daily by everyone involved with the trainee. A compromise will have to be reached between a method that gains sufficient information to draw a full picture of the trainee's developing performance and the need to run services on a daily basis.

The picture emerging in the UK is based on PMETB's curriculum for the foundation years of postgraduate education and training, the core competency requirements of which are summarised in Table 1 . The assessment system will take a threetiered approach, encompassing educational (not National Health Service) appraisal, assessment and annual review (Postgraduate Medical Education and Training Board, 2005). 
Table 1 Foundation programme core competencies (after Foundation Programme Committee of the Academy of the Medical Royal Colleges, 2005)

Domain
Good clinical care

Maintaining good medical practice

Relationships with patients and communication

Working with colleagues

Teaching and training

Professional behaviour and probity

Acute care

\section{Skills/competencies}

History-taking; patient examination (includes mental state examination); record-keeping; time management; decisionmaking. Understands and applies the basis of maintaining good quality care; ensures and promotes patient safety; knows and applies the principles of infection control; understands and applies the principles of health promotion/public health; understands and applies the principles of medical ethics and relevant legal issues

Self-reflective learning skills; directs own learning; adheres to organisational rules/guidelines; appraises evidence base of clinical practice; employs evidence-based practice; understands the principles of audit

Demonstrates good communication skills

Working in teams; managing patients at the interface of different specialties, including primary care, imaging and laboratory specialties

Understands educational methods; teaches medical trainees and other health professionals

Consistently behaves professionally; maintains own health; self-care

Assessing, managing and treating acutely ill/collapsed/ unconscious or semi-conscious/convulsing/psychotic/toxic patients or patients who have harmed themselves: this includes giving fluid challenge, analgesia and obtaining an arterial sample for blood gas. Recognising own limits and asking for help appropriately; handing over information to relevant staff; taking patients' wishes into consideration; resuscitation of patients, including consideration of advance directives
A thorough discussion of some of the different assessment tools and how they may be used follows. It must be recognised though that this is an area of considerable growth. New tools are being developed and many of these will become part of the collection of evidence documented in individual portfolios in years to come.

\section{Assessment tools and methods} The mini-clinical evaluation exercise

The mini-clinical evaluation exercise (mini-CEX) has been adapted from the clinical evaluation exercise (CEX), an instrument designed by the American Board of Internal Medicine for assessing junior doctors at the bedside. The CEX is an oral examination during which the trainee, under the observation of one assessor who is a physician, takes a full history and completes an full examination of a patient to reach a diagnosis and plan for treatment. It takes about $2 \mathrm{~h}$ to complete. The physician gives immediate feedback to the trainee and documents the encounter. Later the trainee gives the evaluator a written record of the patient's work-up. The CEX has limited generalisability (Kroboth et al, 1992; Noel et al, 1992) because it is limited to one patient and one assessor, making it a snapshot view, vulnerable to rater bias.

The mini-CEX (Norcini et al, 2003) involves several assessments, each of 20 min duration, that are conducted at intervals during training. Each clinical encounter is selected to focus on areas and skills selected from the foundation curriculum (Foundation Programme Committee of the Academy of the Medical Royal Colleges, 2005) (see Table 1). A trainee will undertake six to eight mini-CEXs over the course of foundation-level training (Table 1). Each assessment will be rated by a single but different examiner and will assess skills not previously examined. Rather than completing a full history and examination the trainee is asked to conduct a focused interview and examination, for example to assess the suicidal intent of a patient. Assessment takes place in settings in which doctors would normally see patients (out-patient clinics, on 
the wards) and immediate direct feedback is given to the trainee.

The mini-CEX has been demonstrated to have good reproducibility (Norcini et al, 1995), validity and reliability (Kroboth et al, 1992; Durning et al, 2002; Kogan et al, 2003) in general medicine.

The number of mini-CEXs required in higher specialist training is yet to be determined. The studies on reproducibility suggest that, for a given area of performance, at least four assessments are needed if the trainee is doing well and more than four if performance is marginal or borderline (Norcini et al, 1995).

As yet the mini-CEX has not been evaluated specifically with psychiatric trainees. Early experience suggests that the practical planning (e.g. coordinating diaries to book the session and to give time to find a case that meets the criteria) should be done well in advance. The time required for an assessment stated in the foundation-years curriculum (Foundation Programme Committee of the Academy of Medical Royal Colleges, 2005) may be an underestimate and resources are required in terms of time and patients. Importantly, the examiners will require extensive training.

\section{The standardised patient examination}

A standardised patient is a person who is trained to take the role of a patient in a way that is similar and reproducible for each encounter with different trainees. Hence they present in an identical way to each trainee. The standardised patient may be an actor, an asymptomatic patient or a patient with stable, abnormal signs on examination. Standardised patients can be employed for teaching history-taking, examination, communication skills and interpersonal skills. They can also be used in the assessment of these skills. The advantages of using standardised patients are that the same clinical scenario is presented to each trainee (reliability) and they enable direct observation of clinical skills (face validity). Feedback can be immediate and can also be given from the point of view of the patient - although the standardised patient would need to be trained to do this in a constructive manner.

Using standardised patients has high face validity. Reliability varies from 0.41 to 0.85 (Holmboe \& Hawkins, 1998), increasing with more cases, shorter testing times and less complex cases. The reliability of this type of assessment is better when assessing history-taking, examination and communication skills than for clinical reasoning or problemsolving. Standardised patients have been used in multi-station exams such as OSCEs, where trainees perform focused tasks at a series of stations.
Standardised patients have been used as a means of integrating the teaching and learning of interpersonal skills with technical skills and giving direct feedback to trainees. If the student-patient encounter is video recorded the student can subsequently review the tape as an aid to learning (Kneebone et al, 2005). Video recordings can also be used as part of a trainee's assessment, for example by enabling multiple raters to assess the individual (thereby increasing reliability). For workplacebased assessments the standardised patient would be seen in a setting where the candidate normally sees patients.

\section{Case-based discussion}

Case-based discussion is also known as chartstimulated recall examination (Munger, 1995) and strategic management simulation (Satish et al, 2001). The trainee discusses his or her cases with two trained assessors in a standardised and structured oral examination, the purpose of which is to evaluate the trainee's clinical decision-making, reasoning and application of medical knowledge with real patients. The assessors question the trainee about the care provided in predefined areas - problem definition (i.e. diagnosis), clinical thinking (interpretation of findings), management and anticipatory care (treatment and care plans) (Southgate et al, 2001). The trainee is rated according to a prescribed schedule; a single examination takes about $10 \mathrm{~min}$, and there will be about six to eight examinations during the course of training. The assessors (two independent raters) visit the trainee's place of work and the patient's notes/trainee's documentation are used as the focus of the exam.

Patients must be selected to form a representative sample. The trainee's performance is determined by combining scores from a series for a pass/fail decision. Reliabilities of between 0.65 and 0.88 have been reported in other medical specialties (Accreditation Council for Graduate Medical Education \& American Board of Medical Specialties, 2000). Assessors must be trained in how to question and how to score responses.

Case-based discussion is potentially easy to implement but it requires that trainees manage sufficient and sufficiently varied cases to give a valid and reliable sample. Extensive resources and expertise will be required to standardise the examination if it is to be used for 'high-stakes' assessments.

\section{Directly observed procedures}

Directly observed procedures or objective structured assessment of technical skills are similar to the mini-CEX. The process has been developed by 
the Royal College of Physicians to assess practical skills (Wilkinson et al, 2003). Two examiners observe the trainee carrying out a procedural task on a patient and independently grade the performance. This form of assessment lends itself well to medical specialties where the acquisition of practical skills is important. Examples include suturing a wound, taking an arterial blood sample and performing a lumbar puncture.

In psychiatry it is less obvious what we would observe because psychiatrists are less involved with practical procedures. One possibility would be assessing how a trainee administers electroconvulsive therapy (ECT), but this is not frequently prescribed. Alternatively it could be used to assess less concrete skills such as conducting a risk assessment on a patient who has recently taken an overdose or preparing a patient for ECT, explaining the process and obtaining consent. In practice, there could be considerable overlap with the mini-CEX.

\section{Multisource feedback}

The need for a measure of the humanistic aspects of a doctor's practice is increasingly accepted. Multisource feedback, which is also known as $360^{\circ}$ assessment, consists of measurements completed by many people in a doctor's sphere of influence. Evaluators completing the forms are usually colleagues, other doctors and members of the multiprofessional team. Rarely are patients or families invited to participate. A multisource assessment can be used to provide data on interpersonal skills such as integrity, compassion, responsibility to others and communication; on professional behaviours; and on aspects of patient care and team-working. The data collected are summarised to give feedback.

Raters tend to be more accurate and less lenient when an evaluation is intended to give formative rather than summative assessment (see Box 2 for definitions) (Accreditation Council for Graduate Medical Education \& American Board of Medical Specialties, 2000). A body of published work is emerging (Evans et al, 2004; Baker, 2005) that suggests the clear need to differentiate between a tool to be used in developmental appraisal and a method for identifying poor performance. This differentiation is not always apparent. There must also be a note of caution surrounding the assessment of behaviours that are not directly observable. None the less, multisource feedback can make a vital contribution to the measurement and improvement of an individual doctor's performance.

There remain two practical challenges. First, construction of tools that all potential assessors can use and second, the compilation of the data into a format that can be reported confidentially to the trainee.

\section{Other assessments}

Other assessment tools exist although not strictly for workplace assessments. However, they are worthy of a mention because they have the potential to complement workplace assessments for a complete all-round assessment package.

\section{Written examination}

Although workplace assessments cover the trainees' performance they are weak at assessing their knowledge in a broad sense. This can be done using multiple choice or similar written papers such as those listed in Box 3.

\section{Portfolios}

A portfolio is a collection of material, collated by the individual over time, used to demonstrate their chronological learning, performance and development. It can enable individuals to reflect on their abilities and highlight areas that could be improved to enhance the quality of their practice. There are numerous models for portfolio use. In medicine, portfolios tend to be used both formatively (to improve one's own practice) and summatively (for recertification and revalidation). However, there is little evidence to support their use for the latter.

For portfolios to be a valid and reliable assessment tool trainees need to be well prepared in using them and their content must be uniform. Raters should be well trained and experienced (Roberts et al, 2002). Assessment criteria should be consistent with what doctors are expected to learn. The NHS Appraisal Toolkit (http://www.appraisals.nhs.uk) includes a model portfolio for doctors that covers the areas outlined in the GMC's Good Medical Practice (General Medical Council, 2001) and summarised in Box 4.

The advantage of establishing a portfolio during postgraduate training is that it is flexible and can

\section{Box 3 Written assessments}

Multiple choice questions The candidate has to select the correct response to a stem question or statement from a list of one correct and four incorrect answers (other formats can be used)

Best of five The candidate has to select the most likely answer out of five options, some of which could be correct but one is more likely than the others

Extended matching questions The candidatehas to match one out of several possible answers to each of a series of statements 


\section{Box 4 Summary of the duties of a doctor} registered with the GMC

- Good clinical care

- Maintaining good medical practice

- Partnership with patients

- Working with colleagues and in teams

- Assuring and improving the quality of care

- Teaching and training

- Probity

- Health

(General Medical Council, 2001)

therefore accommodate the individual's varying needs as they proceed along chosen career paths and within different specialties.

\section{The record of in-training assessment}

The record of in-training assessment (RITA) is a portfolio of assessments that are carried out during medical training. It used throughout UK postgraduate medical training, and is mandatory for specialist registrars and is being introduced for senior house officers. The purpose of this record is to determine whether or not an individual has completed each training period satisfactorily and can proceed with training and ultimately enter the specialist register. The Guide to Specialist Registrar Training (Department of Health, 1998) describes the RITA system, but the generality of its recommendations has resulted in widespread variation between regions and specialties.

A RITA is an assessment record, not an assessment in itself, and it currently relies on in-service assessments that are almost always retrospective and either lack evidence or use unsubstantiated evidence of competence (Postgraduate Medical Education and Training Board, 2005). The clear opportunity now arises to clarify and strengthen a RITA-type system, as new workplace-based assessment processes begin to yield recordable evidence of performance.

\section{Resource requirements: the professionalisation of training}

The implementation of an assessment structure that adds value to the system for training and accreditation of doctors will require urgent attention to resources. Extensive assessor training will be needed to ensure uniformity of assessment at a local level. As most consultants will be expected to carry out workplace assessments, training that requires assessors to take professional leave may pose a particular problem. One long-term solution might be to incorporate 'training the trainers' into the curriculum, so that doctors in training are themselves learning to become the trainers of the future (Vassilas et al, 2003).

Unfortunately, the financial implications of producing robust assessment systems have yet to be calculated. Increasing the level of trainee supervision and assessment will almost certainly result in some reduction in time available for service commitment, although this may be difficult to quantify in advance.

The schedule of assessments selected will be mapped to the foundation years' curriculum (Foundation Programme Committee of the Academy of the Medical Royal Colleges, 2005) in a blueprint. The Royal College of Physicians has published a model based on Good Medical Practice and the PMETB Standards for Curricula (2004b) that may serve as an exemplar (Joint Committee on Higher Medical Training, 2003). It will be necessary to establish clear standards for the process and outcome of assessment and then to monitor those standards across programmes. The obvious danger will be that standards will not be equivalent across the UK and abroad.

In a system that relies on assessment by senior colleagues it is likely that very few trainees will be deemed unsatisfactory. There will remain a need for another (national, independent) assessment, perhaps near the end of specialist training. For trainees who continue to perform unsatisfactorily a robust system for career counselling will be required.

\section{Conclusions}

Currently, workplace-based assessment is underutilised and the way in which it is implemented and documented, principally through the RITA, is variable and often of poor standard. Assessors may be reluctant to make unfavourable judgements and may pass trainees by default, in the absence of real evidence. However, it is expected that workplacebased assessment will become increasingly common.

By its very nature workplace-based assessment has the advantage of high face validity, although its predictive validity is currently unknown (as is the case for almost all existing postgraduate medical examinations). Many of the specialties for which the assessments were designed have published reliability data, but there is a pressing need for comparable data in the field of psychiatry. Without evidence specific to our specialty, the public and professionals may well lack confidence in decisions based on the workplace-based assessment process. 
Workplace-based assessment is an excellent source of information for teaching, educational supervision and feedback, as well as providing evidence of satisfactory (or unsatisfactory) progress and achievement. The system is founded on selfdirected learning, so that the trainee schedules each assessment. Trainees will require a good level of organisation and motivation to achieve this. The significant benefit for trainees is the receipt of immediate and constructive feedback.

One of the most likely consequences of the contemporary reforms in postgraduate medical education is an increase in the amount of educational activity in the workplace and this must be properly underpinned if the opportunities offered are to be realised. Each college or specialty must develop a strategy involving workplace-based assessment and examinations of knowledge and clinical skills relating to the entire training period. The tools that we have described here are some of the basic building blocks from which the curriculum and assessment strategy of the future will be constructed.

\section{Declaration of interest}

The views expressed are personal and do not represent the views of the Royal College of Psychiatrists.

\section{References}

Accreditation Council for Graduate Medical Education \& American Board of Medical Specialties (2000) Toolbox of Assessment Methods. Version 1.0. Chicago, IL: ACGME \& ABMS.

Baker, R. (2005) Can poorly performing doctors blame their assessment tools? BMJ, 330, 1254.

Broadfoot, P. (1996) Assessment and Learning: Power or Partnership? New York: John Wiley \& Sons.

Department of Health (1998) Guide to Specialist Registrar Training. London: Department of Health.

Durning, S. J., Cation, L. J., Markert, R. J., et al (2002) Assessing the reliability and validity of the mini-clinical evaluation exercise for internal medicine residency training. Academic Medicine, $77,900-904$.

Evans, R., Elwyn, G. \& Edwards, A. (2004) A review of instruments for peer assessment of physicians. BMJ, 328, 1240-1243.

Foundation Programme Committee of the Academy of the Medical Royal Colleges (2005) Curriculum for the Foundation Years in Postgraduate Education and Training. London: TSO (The Stationery Office). http://www.dh.gov.uk/ assetRoot/04/10/76/96/04107696.pdf

General Medical Council (1993) Tomorrow's Doctors. London: GMC.

General Medical Council (2001) Good Medical Practice (3rd edn). London: GMC.

Holmboe, E. S. \& Hawkins, R. E. (1998) Methods for evaluating the clinical competence of residents in internal medicine. Annals of Internal Medicine, 129, 42-48.

Joint Committee on Higher Medical Training (2003) Higher Medical Training Generic Curriculum. London: JCHMT.

Kneebone, R. L., Kidd, J., Nestel, D., et al (2005) Blurring the boundaries: scenario-based simulation in a clinical setting. Medical Education, 39, 580-587.
Kogan, J. R., Bellini, L. M. \& Shea, J. A. (2003) Feasibility, reliability and validity of the Mini-Clinical Evaluation Exercise (mCEX) in a medicine core clerkship. Academic Medicine, 78, s33-s35.

Kroboth, F. J., Hanusa, B. H., Parker, S., et al (1992) The inter-rater reliability and internal consistency of a clinical evaluation exercise. Journal of General Internal Medicine, 7, 174-179.

Miller, G. E. (1990) The assessment of clinical skills/competence/ performance. Academic Medicine, 9, s63-s67.

Munger, B. S. (1995) Oral examinations. In Recertification: New Evaluation Methods and Strategies (eds E. L. Mancall \& P. G. Bashbrook), pp. 39-42. Evanston, IL: American Board of Medical Specialties.

Newble, D. \& Cannon, R. (2001) A Handbook for Medical Teachers. Dordrecht: Kluwer Academic Press.

Noel, G. L., Herbers, J. E. Jr., Caplow, M. P., et al (1992) How well do internal medicine faculty members evaluate the clinical skills of residents? Annals of Internal Medicine, 117, 757-765.

Norcini, J. J., Blank, L. L., Arnold, K. A., et al (1995) The miniCEX. A preliminary investigation. Annals of Internal Medicine, 123, 295-299.

Norcini, J., Blank, L. Duffy, D., et al (2003) The mini-CEX: a method for assessing clinical skills. Annals of Internal Medicine, 138, 476-481.

Postgraduate Medical Education and Training Board (2004a) Principles for an Assessment System for Postgraduate Medical Training. London: PMETB.

Postgraduate Medical Education and Training Board (2004b) Standards for Curricula. London: PMETB.

Postgraduate Medical Education and Training Board (2005) Workplace Based Assessment. A Paper from the PMETB Workplace Based Assessment Subcommittee: January 2005. London: PMETB.

Roberts, C., Newble, D. I. \& O'Rourke, A. J. (2002) Portfolio-based assessments in medical education: are they valid and reliable for summative purposes? Medical Education, 36, 899-900.

Rowntree, D. (1987) Assessing Students: How Shall We Know Them? London: Kogan Page.

Satish, U., Streufert, S., Marshall, R., et al (2001) Strategic management simulations is a novel way to measure resident competencies. American Journal of Surgery, 181, 557-561.

Stobart, G. \& Gipps, C. (1997) Assessment: A Teacher's Guide to the Issues (3rd edn). London: Hodder and Stoughton Educational

Southgate, L., Cox, J., David, T., et al (2001) The General Medical Council's performance procedures: peer review of performance in the workplace. Medical Education, 35, 9-19.

Vassilas, C. A., Brown, N., Wall, D., et al (2003) 'Teaching the teachers' in psychiatry. Advances in Psychiatric Treatment, 9, 308-315.

Wilkinson, J., Benjamin, A. \& Wade, W. (2003) Assessing the performance of doctors in training. BMJ Career Focus, 327, s91-s92.

\section{MCQs}

1 Assessments:

a do not influence the content of what a trainee learns

b should have high reliability and validity

c are the same as appraisals

$\mathrm{d}$ are useful if they are used only to assess a trainee's knowledge

e are better when they are summative.

2 Workplace-based assessments:

a will not be time consuming for the assessors

b aim to measure what a doctor does in day-to-day practice

c can include assessments by patients

$\mathrm{d}$ include multiple choice questions as a form of assessment

e should be organised by the trainee. 
3 Multisource feedback is useful for assessing:

a a trainee's team-working skills

b a trainee's ability to do a lumbar puncture

c a trainee's ability to communicate effectively

d whether or not a trainee can tie their shoelaces

e a trainee's ability to relate to others.

\section{Portfolios:}

a are ideal for summative assessments

b are a chronological record of a doctor's performance

c enable one to reflect on one's own practice

d enable one to identify areas of weakness in one's own practice

e require that training be provided in their use and assessment.

5 Direct observation of procedural skills:

a requires that the assessors be trained

b can be adapted to assess skills in psychiatric practice c has been developed more for other medical specialties

$\mathrm{d}$ is highly relevant to psychiatric practice

e could be used to assess obtaining patient consent to treatment.

\begin{tabular}{|llllll|}
\hline MCQ answers & & & & \\
1 & & 2 & & & \\
a F & a F & a T & a F & a T \\
b T & b T & b F & b T & b T \\
c F & c T & c T & c T & c T \\
d F & d F & d F & d T & d F \\
e F & e T & e T & e T & e T \\
& & & & &
\end{tabular}

\section{Hazards ahead?}

INVITED COMMENTARY ON:

ASSESSING PROFESSIONAL AND CLINICAL COMPETENCE

Nick Rose

Abstract In the most radical (and politically driven) changes to the National Health Service since it was founded, the training and assessment of doctors will focus more on what they do than on what they know. The UK's lack of tools to assess doctors' performance in the workplace has caused the Postgraduate Medical Education and Training Board to turn to US workplace assessement tools developed for medical practice. These have not been designed or evaluated for application to psychiatric practice, nor do they allow for independent evaluation. It remains unclear how workplace assessment in psychiatric training will tie in with national examinations such as the Royal College of Psychiatrists' membership examination (the MRCPsych); nor is it clear whether service users are yet to have a say in such an important matter as the training of their doctors.

Medical education has finally got political, a fact that informs Brown \& Doshi's analysis but is never named (Brown \& Doshi, 2006, this issue). What they omit to mention is that doctors and the medical Royal Colleges are positioning themselves to deal with some of the most radical changes since the National Health Service (NHS) began, involving the restructuring of what we expect of doctors, the redefining of boundaries with other healthcare staff and some redistribution of power from the Royal Colleges to the newly created Postgraduate Medical Education and Training Board (PMETB). Changing the medical profession has been on the political agenda at least since the Thatcher era, but

Nick Rose is a consultant psychiatrist (Littlemore Mental Health Centre, Sandford Road, Littlemore, Oxford OX4 4XN, UK. E-mail: nick. rose@oxmhc-tr.nhs.uk) and honorary senior lecturer at the University of Oxford. He acts as Regional Advisor for Psychiatry with the Oxford Deanery and is a trainer on the Foundation Year 2 pilot scheme. 